\title{
A Novel 2-Lead to 12 Lead ECG Reconstruction Methodology for Remote Health Monitoring Applications
}

\author{
Naresh Vemishetty ${ }^{1,2}$, Vishnuvardhan Gundlapalle ${ }^{1,2}$, Amit Acharyya ${ }^{1,2^{*}}$, Bhudeb Chakravarti ${ }^{2}$ \\ ${ }^{1}$ Indian Institute of Technology-Hyderabad, India \\ ${ }^{2}$ Sense Health Technologies Pvt. Ltd, Hyderabad, India
}

\begin{abstract}
Standard clinical 12-lead ECG is the setup most commonly used by the doctors for the reliable diagnosis. However, placing all the electrodes on the body will be inconvenient and affect the daily activities of the patient. In addition, transmitting all the lead information will require high power and large memory in remote ECG monitoring. Accounting this, we propose here a novel 2 to 12 lead reduction system for remote ECG monitoring applications. In the proposed method, the coefficient values of all the leads are generated as the first step using the least-square fit method and heart-vector projection. For the 12 lead ECG reconstruction, lead I and V2 are taken as the basis leads. As a part of the reconstruction, Lead II is derived initially from these two basis leads (Lead I, V2) using the HVP computation. After Lead II derivation, these three (I, II, V2) leads acts as basis leads and will derive remaining leads $(V 1, V 3, V 4, V 5, V 6)$ by repeating the HVP computation. Three categories Healthy Control (HC), Bundle Branch Block (BBB) and, Myocardial Infarction (MI) were taken from PTBDB for the proposed work. $R^{2}$ statistics, correlation and regression coefficients were used to evaluate the performance, the mean values of the stated performance metrics obtained were $91.94 \%$, 0.957, 0.921 for $H C, 85.81 \%, 0.920,0.856$ for $B B B$ and $81.42 \%, 0.889,0.820$ for MI respectively.
\end{abstract}

\section{Introduction}

Cardiovascular diseases (CVD) are one of the prime causes of human mortality worldwide [1] and therefore tremendous research has been done for the classification and prevention of CVD [2]. The Electrocardiogram (ECG) assists cardiologists in the diagnosis of heart conditions. In recent years, advancement in technology has made remote ECG monitoring possible with considerably small equipment [3]. There are multiple ways to acquire the ECG, but the standard clinical 12-lead acquisition is the setup most commonly used by the doctors for the reliable diagnosis. Consequently, 12-lead ECG monitoring is limited to short timespans while remote long-term ECG monitoring relies on two-lead or three-lead ECGs, which provide limited information [4] on heart condition. In clinical practice, continuous recording of all the standard 12 lead ECG from the patient is often not possible for longer duration. For the remote ECG monitoring, placing all the 10 electrodes on patient body with all connecting wires will create inconvenient and it will affect the daily activities. In addition, transmitting all the lead information will require high power and large memory. Thus to minimize these constraints it is essential to get 12 lead system from reduced lead sets which is important for improving patient comfort during long-term monitoring. However, it will have a significant impact on patient psychology. These bottlenecks have motivated us to propose a novel 2 to 12 lead reconstruction system. Hence, the proposed work is focussed on lead reconstruction using Lead I and V2 as the basis leads.

There are few research articles which are available for reduced 3 lead to 12 lead ECG reconstruction [5]. Linear regression is one of the methods [6], there are few nonlinear methods which includes artificial neural networks (ANN) [7] and General Vector Machine (GVM) [8] models. Other investigations involving the reconstruction of precordial leads are [9-10]. But these methods are computationally very intensive compared to that of linear methods for the remote CVD monitoring as the device should be portable.

There is a scope for further reducing the usage of 3 lead set to 2 lead set to reconstruct the 12 lead ECG data, which helps in the reduction of electrodes usage from 5 to 4 while collecting the ECG data. Accounting this, we have proposed a novel low complex 2 lead to 12 lead methodology for remote health monitoring applications. Reduction from the state of the art 3 leads to proposed 2 lead minuscule from the theoretical perspective. The reconstruction of leads in this work depends on the coefficients generated and it is personalized. The paper is organized as follows, section 2 describes the proposed methodology and its detailed explanation. Section 3 shows the analytic results and discussions, section 4 concludes the proposed work. 


\section{Proposed Methodology}

\subsection{Database and Pre-processing}

ECG database sampled at $1 \mathrm{KHz}$ frequency has been taken from the Physionet's PTBDB. Three categories Healthy Control (HC), Bundle Branch Block (BBB), and Myocardial Infarction (MI) are chosen to validate the proposed methodology. $\mathrm{HC}$ consists of 80 patient's data, BBB consists of 16 patient's data and MI consists of 333 patient's data, each patient data holds 1-minute recording. As a part of pre-processing, artifacts are removed from the raw ECG signal by passing through $4^{\text {th }}$ order butter worth band pass filter having cut-off frequencies as $0.05 \mathrm{~Hz}$ and $40 \mathrm{~Hz}$, as most of the ECG signal frequencies lies in that range. Median filter is used to estimate baseline which is then subtracted from the filtered signal. The pre-processed data is then used for further processing throughout the work.

\subsection{Proposed 2 to 12 lead reconstruction methodology}

The noise free 12 lead ECG signals obtained from the pre-processing block are then used for generating the personalized coefficients using Least Square (LS) fit method and Heart-Vector Projection (HVP) [5] theory as shown in Figure 1. The reduced 2 lead system along with the generated personalized coefficients are used to reconstruct the missing leads as shown in Figure 2. $\mathrm{R}^{2}$ statistics, correlation $\left(r_{x}\right)$ and regression $\left(b_{x}\right)$ coefficients are used to evaluate the reconstruction performance.

\begin{tabular}{|c|c|c|}
\hline Lead I & \multirow{9}{*}{$\begin{array}{l}\text { Reconstruction } \\
\text { Coefficient } \\
\text { Generation using } \\
\text { Least Square fit } \\
\text { method }\end{array}$} & RC of lead I \\
\hline Lead II & & RC of lead II \\
\hline Lead III & & RC of lead III \\
\hline V1 & & $\mathrm{RC}$ of $\mathrm{V} 1$ \\
\hline V2 & & $\mathrm{RC}$ of $\mathrm{V} 2$ \\
\hline V3 & & $\mathrm{RC}$ of $\mathrm{V} 3$ \\
\hline V4 & & RC of V4 \\
\hline V5 & & $\mathrm{RC}$ of $\mathrm{V} 5$ \\
\hline V6 & & $\mathrm{RC}$ of $\mathrm{V} 6$ \\
\hline
\end{tabular}

Figure 1. Generation of the coefficients for all the leads with the existing data.

\section{Personalized coefficients generation using LS fit method and $\mathrm{HVP}$ theory}

The potential at any point of the body is the projection of the heart vector $(\vec{H})$ on the lead vector $(\vec{L})$ placed on the body as described in equation 1 .

$$
\mathrm{V}=\vec{H} \cdot \vec{L}=a_{1} \mathrm{X}+b_{1} \mathrm{Y}+c_{1} \mathrm{Z}
$$

Where $\vec{H}=\mathrm{X} \hat{\imath}+\mathrm{Y} \hat{\jmath}+\mathrm{Z} \hat{k}, \vec{L}=a_{1} \hat{\imath}+b_{1} \hat{\jmath}+c_{1} \hat{k}$, this linear model will be helpful to obtain the potential at any point using leads apart from the heart vector components [5].

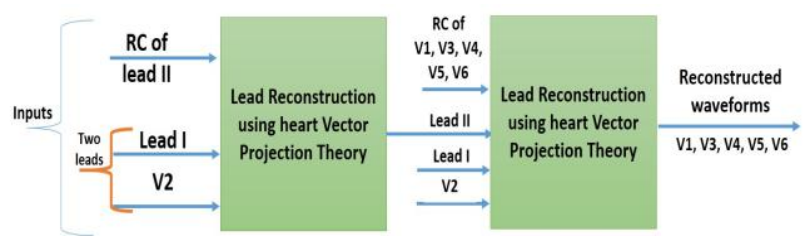

Figure 2. Reduced lead system to reconstruct the 12 lead system using 2 lead ECG.

In proposed method, Lead I and V2 are taken as the basis leads to reconstruct the missing leads (II, V1, V3, V4, V5 and V6). Lead III, aVR, aVL and aVF can be reconstructed using the standard procedure (Lead III=Lead II-Lead I) using Lead I and II. Hence, these leads are not reconstructed using the proposed method. Firstly, we will derive lead II using these two basis leads (I, V2) in the proposed method. Lead II can be represented as shown in equation (2) using HVP computation.

$$
I I=a_{1} I+b_{1} V 2
$$

These personalized coefficients $\left(a_{1}, b_{1}\right)$ are generated using LS fit method as shown in equation (3).

$$
\left[\begin{array}{l}
a_{1} \\
b_{1}
\end{array}\right]=\left[\begin{array}{cc}
\sum I^{2} & \sum I \cdot V_{2} \\
\sum I . V_{2} & \sum V_{2}^{2}
\end{array}\right]^{-1}\left[\begin{array}{c}
\sum I I . I \\
\sum I I . V_{2}
\end{array}\right]
$$

After obtaining Lead II, we use these three leads (I, II, V2) as the basis leads and will derive the remaining leads (V1, $\mathrm{V} 3$ to V6). Lead $\mathrm{V}_{\mathrm{i}}$ can be represented as shown in equation (4) using HVP computation where lead $V_{i}$ can be any one of the missing leads (V1, V3 to V6).

$$
V_{i}=x_{i} I+y_{i} I I+z_{i} V 2
$$

These personalized coefficients $\left(x_{i}, y_{i}, z_{i}\right)$ are generated using LS fit method as shown in equation (5).

$$
\left[\begin{array}{l}
x_{i} \\
y_{i} \\
z_{i}
\end{array}\right]=\left[\begin{array}{ccc}
\sum I^{2} & \sum I . I I & \sum I \cdot V_{2} \\
\sum I . I I & \sum I I^{2} & \sum I I . V_{2} \\
\sum I . V_{2} & \sum I I . V_{2} & \sum V_{2}{ }^{2}
\end{array}\right]^{-1}\left[\begin{array}{c}
\sum V_{i} \cdot I \\
\sum V_{i} . I I \\
\sum V_{i} \cdot V_{2}
\end{array}\right]
$$

2 to 12 lead reconstruction without lead II derivation as the first step is also possible, which would require only one computation of HVP. However, the accuracy of reconstructed wave is less as per the statistical analysis. Hence, to increase the accuracy levels of the outcome, we have used two HVP computations as shown in the Figure 2 to reconstruct rest of the leads. Table 2 shows the pseudo code of the proposed methodology 

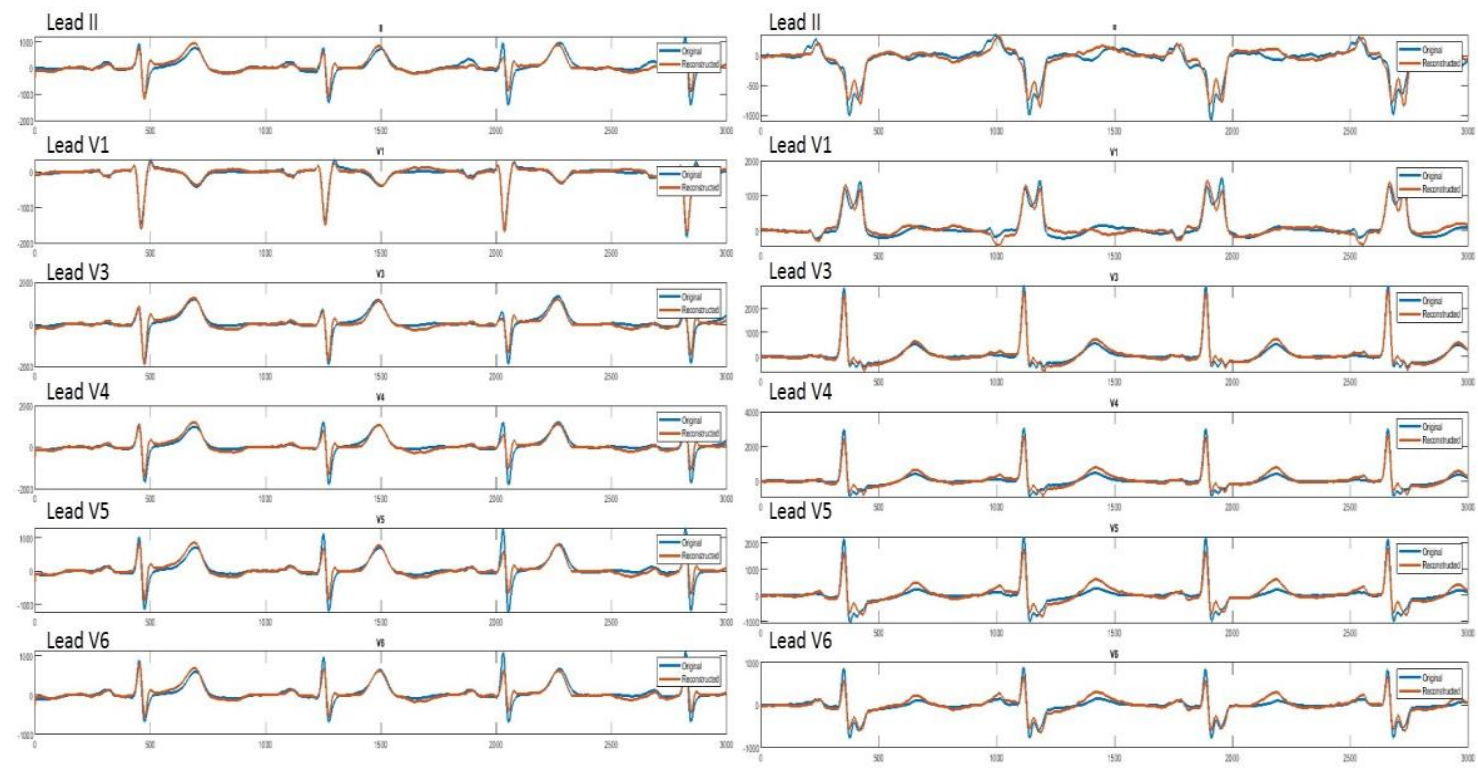

Figure 3. Comparison analysis of original (blue) and reconstructed (red) ECG signals.

Table 1. Performance results of 2 to 12 lead reconstruction

\begin{tabular}{|c|c|c|c|c|c|c|c|c|c|}
\hline $\begin{array}{c}\text { Reconstructed } \\
\text { Leads }\end{array}$ & \multicolumn{3}{|c|}{ Healthy Control (80) } & \multicolumn{3}{c|}{ Bundle Branch Block (16) } & \multicolumn{3}{c|}{ Myocardial Infarction (333) } \\
\hline & $R^{2}$ & $r_{x}$ & $b_{x}$ & $R^{2}$ & $r_{x}$ & $b_{x}$ & $R^{2}$ & $r_{x}$ & $b_{x}$ \\
\hline II & 88.21 & 0.935 & 0.882 & 78.18 & 0.874 & 0.782 & 63.17 & 0.768 & 0.632 \\
\hline V1 & 91.77 & 0.955 & 0.918 & 88.84 & 0.937 & 0.888 & 92.59 & 0.960 & 0.923 \\
\hline V3 & 95.77 & 0.978 & 0.958 & 93.79 & 0.968 & 0.938 & 93.97 & 0.968 & 0.940 \\
\hline V4 & 90.81 & 0.950 & 0.908 & 84.71 & 0.918 & 0.847 & 82.98 & 0.904 & 0.830 \\
\hline V5 & 92.97 & 0.962 & 0.930 & 82.51 & 0.903 & 0.825 & 78.29 & 0.871 & 0.783 \\
\hline V6 & 92.11 & 0.960 & 0.927 & 86.82 & 0.921 & 0.856 & 77.54 & 0.865 & 0.814 \\
\hline Average & $\mathbf{9 1 . 9 4}$ & $\mathbf{0 . 9 5 7}$ & $\mathbf{0 . 9 2 1}$ & $\mathbf{8 5 . 8 1}$ & $\mathbf{0 . 9 2 0}$ & $\mathbf{0 . 8 5 6}$ & $\mathbf{8 1 . 4 2}$ & $\mathbf{0 . 8 8 9}$ & $\mathbf{0 . 8 2 0}$ \\
\hline
\end{tabular}

\section{Performance evaluation metrics}

$\mathrm{R}^{2}$ statistics, correlation $\left(\mathrm{r}_{\mathrm{x}}\right)$ coefficient and regression $\left(b_{\mathrm{x}}\right)$ coefficients are used to evaluate the reconstruction performance. Formulas for these mentioned metrics are given by equations (5), (6) and (7) respectively.

$R^{2}=\left\{1-\frac{\sum[\text { Derived }(\text { sample } i)-\text { Measured }(\text { sample } i)]^{2}}{\sum[\text { Measured }(\text { sample } i)]^{2}}\right\} \times$

100

$r_{x}=\left\{\frac{\sum[\text { Derived }(\text { sample } i) \times \text { Measured }(\text { sample } i)]}{\left[\sum(\text { Derived }(\text { sample } i))^{2} \times \sum(\text { Measured }(\text { sample } i))^{2}\right]^{\frac{1}{2}}}\right\}$ $b_{x}=\left\{\frac{\sum[\text { Derived }(\text { sample } i) \times \text { Measured }(\text { sample } i)]}{\sum[\text { Measured }(\text { sample } i)]^{2}}\right\}$

Table 1 presents the mean values of $\mathrm{R}^{2}$ statistics, correlation $\left(r_{x}\right)$ and regression $\left(b_{x}\right)$ coefficients for the three categories $\mathrm{HC}, \mathrm{BBB}$ and MI. The mean values of the stated performance metrics obtained were $91.94 \%, 0.957 \%$, $0.921 \%$ for $\mathrm{HC}, 85.81 \%, 0.920 \%, 0.856 \%$ for $\mathrm{BBB}$ and $81.42 \%, 0.889 \%, 0.820 \%$ for MI respectively. From the Table 1 , it is evident that the maximum reconstruction performance obtained for lead V3 for all the categories while the minimum reconstruction performance obtained for lead II. And also the results show that $\mathrm{HC}$ reconstruction performance is greater than that of $\mathrm{BBB}$ and MI. This indicates that the accuracy of reconstruction depends on many factors which affect the values of personalized coefficients and it can be interpreted that the performance in incremented and decremented due to the 
variation in the placement of electrodes while recording the ECG data, as the electrodes placement changes for every recording on the body. Other factors like age, sex, size, shape, fat distribution and any arrhythmias are less likely to change the coefficient variation. Figure 3 shows the originals vs reconstructed signal plots. It illustrates that most of the clinical features are retained in the reconstructed signal for all the missing leads.

Table 2. Pseudo code for 2 to 12 lead reconstruction

1) Apply filtering on the ECG signal to remove the noise and baseline wandering

2) Generate reconstruction coefficients for all leads as shown in Figure 1.

3) Consider Lead I and Lead V2 as the basis leads

4) Using HVP, reconstruct Lead II using Lead I and V2 as shown in equation 1.

5) Lead III, aVR, aVL and aVF are derived using the Lead I and the extracted Lead II using the standard procedure.

6) Consider Lead I, II and Lead V2 as the basis leads

7) Using HVP, reconstruct each missing lead using the equation 5 .

8) Plot the reconstructed and original waves for the comparison

9) Perform $R^{2}$ statistics, correlation coefficient and regression to evaluate the proposed method

10) The same procedure is followed for the subjects of different categories consider for the proposed method.

\section{Conclusion}

In this paper, a novel 2-Lead to 12 lead ECG reconstruction methodology is proposed for remote health monitoring applications. In the proposed method, the coefficient values of all the leads are generated as the first step using the least-square fit method and heart-vector projection. For the 12 lead ECG reconstruction, lead I and V2 are taken as the basis leads in this work. As a part of the reconstruction, Lead II is derived initially from these two basis leads (Lead I, V2) using the HVP computation. After Lead II derivation, the three (I, II, V2) leads acts as basis leads to derive the remaining leads (V1, V3, V4, V5, V6) by repeating the HVP computation. The results show that the proposed methodology is reliable and accurate reconstruction is possible without losing the clinical significant features. As the proposed method is less computationally intensive than the conventional methods, we can implement this technique on edge devices for remote health monitoring applications.
This work is partly supported by Sense Health Technologies private limited and Department of Science \& Technology (DST) under the Internet of Things (IoT) Research of Interdisciplinary Cyber Physical Systems (ICPS) Programme, GOI, New Delhi, with the Project entitled "IOT Based Holistic Prevention and Prediction of CVD (i-PREACT)".

\section{References}

[1] CVD statistics as per WHO." [Online]. Available: http://www.who.int/cardiovascular_diseases/en/

[2] Vemishetty, Naresh, et al. "Low power personalized ECG based system design methodology for remote cardiac health monitoring." IEEE Access 4, pp. 8407-8417, 2016.

[3] Puddu,Paolo Emilio, et al. "A clinician's view of nextgeneration remote healthcare system." Systems design for remote healthcare. Springer, New York, pp. 1-30, 2014.

[4] Hoekem, R., G. J. H. Uijen, and A. Van Oosterom. "On selecting a body surface mapping procedure." Journal of Electrocardiology 32.2, pp. 93-101, 1999.

[5] Maheshwari, Sidharth, Amit Acharyya, Pachamuthu Rajalakshmi, Paolo Emilio Puddu, and Michele Schiariti. "Accurate and reliable 3-lead to 12-lead ECG reconstruction methodology for remote health monitoring applications." IRBM 35, vol. 6, pp. 341-350, 2014.

[6] Modre, Robert, et al. "Cardiac anisotropy: Is it negligible regarding noninvasive activation time imaging?." IEEE Transactions on Biomedical Engineering 53.4, pp. 569-580, 2006.

[7] Atoui, H., J. Fayn, and P. Rubel. "A neural network approach for patient-specific 12-lead ECG synthesis in patient monitoring environments." Computers in Cardiology, IEEE, 2004.

[8] Xu, Zijian, Rui Zhou, Yunshan Cao, Binbin Yong, Xin Wang, and Qingguo Zhou. "Reconstruction of 12-Lead Electrocardiogram Based on GVM." In 2018 Sixth International Conference on Advanced Cloud and Big Data (CBD), pp. 275-280. IEEE, 2018.

[9] Nelwan, Stefan P., Jan A. Kors, Simon H. Meij, Jan H. van Bemmel, and Maarten L. Simoons. "Reconstruction of the 12lead electrocardiogram from reduced lead sets." Journal of electrocardiology vol. 37, pp. 11-18, 2004.

[10] Feild, Dirk Q., Sophia H. Zhou, Eric D. Helfenbein, Richard E. Gregg, and James M. Lindauer. "Technical challenges and future directions in lead reconstruction for reduced-lead systems." Journal of electrocardiology 41, no. 6, pp. 466-473, 2008.

Address for correspondence.

Name. Amit Acharyya

Full postal address. Associate Professor, Room No: 505,

Academic Block-C, Department of

Electrical Engineering, Indian Institute of

Technology, Hyderabad, Kandi, Hyderabad,

Telangana. INDIA. Pin Code: 502285

E-mail address. amit_acharyya@iith.ac.in,

Web site: http://www.iith.ac.in/ amit_acharyya/

\section{Acknowledgements}

